\title{
Caracterización del Bosque Seco Tropical Remanente en el Valle de Agalta, Honduras
}

\author{
José Manuel Mora ${ }^{1}$, Mario Roberto Espinal ${ }^{2}$, Lucía Isabel López ${ }^{3}$ y Banny Omar Quezada ${ }^{4}$
}

Resumen. Identificamos 20 fragmentos de Bosque Seco Tropical en el valle de Agalta, Olancho, Honduras. Caracterizamos la estructura de la vegetación de los fragmentos y la dominancia e importancia relativa de las especies vegetales y otros parámetros dasométricos de su organización horizontal y vertical. Identificamos 265 especies de plantas, distribuidas en 78 familias y 223 géneros. El hábito dominante fue las hierbas (81 especies) seguido por los árboles (69 especies). Las familias más comunes fueron las leguminosas (Fabaceae) (36 especies), las orquídeas (17 especies) y las asteráceas (13 especies). Las asociaciones vegetales del valle de Agalta se mezclan con predominancia de los sistemas productivos antropogénicos. La presencia de ciertas especies vegetales sugiere que en el pasado cercano existieron asociaciones más húmedas en el valle. En la actualidad existe el bosque deciduo bajo y el bosque de pino-encino. El biscuite (Acacia deamii) tuvo la abundancia relativa más alta, seguido por el guamuche (Pithecelobium dulce). Esto puede ser el resultado de los rebrotes de las plantas existentes del bosque original. En el bosque de pino-encino dominaron las fagáceas Quercus sapoetifolia y Quercus segoviensis, con una contribución importante del nance (Byrsonima crassifolia, Malpighiaceae). Existe cierta conectividad entre algunos fragmentos de bosque seco tropical en Agalta a través de la vegetación remanente de los bosques rivereños. Lo anterior puede ser de vital importancia para la fauna del valle, en particular del colibrí esmeralda (Amazilia luciae), la única ave endémica de Honduras y clasificada como en peligro de extinción. El Bosque Seco Tropical es una de las zonas de vida menos representada en las áreas protegidas de Honduras a pesar de sus endemismos y la biodiversidad en general que contiene.

Palabras clave: Bosque deciduo bajo, bosque de pino-encino, colibrí esmeralda, fragmentos de bosque, Índice de Valor de Importancia.

\section{Characterization of the Remaining Tropical Dry Forest of the Agalta Valley, Honduras}

\begin{abstract}
We identify 20 fragments of Tropical Dry Forest in the Agalta Valley, Olancho, Honduras. We characterize the vegetation structure of the fragments and the dominance and relative importance of plant species and other dasometric parameters of their horizontal and vertical organization. We identified 265 plant species, distributed in 78 families and 223 genera. The dominant habit was herbs ( 81 species) followed by trees (69 species). The most common families were Fabaceae (36 species), Orchidaceae (17 species) and Asteraceae (13 species). Plant associations at Agalta Valley mixed with predominance of anthropogenic production systems. The presence of certain plant species suggests that in the near past wetter associations existed in the valley. Currently there exist the low deciduous forest and the pine-oak forest. Biscuite (Acacia deamii) had the highest relative abundance, followed by guamuche (Pithecelobium dulce). This may be the result of regrowth of plants remaining from the original forest. The dominant species at the pine - oak forest were the oaks Quercus sapoetifolia and Quercus segoviensis, with a significant contribution of the nance (Byrsonima crassifolia, Malpighiaceae). There is some connectivity between forest fragments at Agalta through the remaining vegetation of riverine forests. This can be of vital importance to wildlife in the valley, including the emerald hummingbird (Amazilia luciae), the only endemic bird species of Honduras and classified as endangered. The Tropical Dry Forest is one of the life zones underrepresented in the protected area system of Honduras despite its high endemism and biodiversity in general it contains.
\end{abstract}

Key words: Emerald hummingbird, forest fragments, Importance Value Index, low deciduous forest, pine-oak forest.

\footnotetext{
${ }^{1}$ Instituto Internacional en Conservación y Manejo de Vida Silvestre (ICOMVIS), Universidad Nacional (UNA), Heredia, Costa Rica. Correo electrónico josemora07@gmail.com

${ }^{2}$ Investigador Asociado, Centro Zamorano de Biodiversidad, Escuela Agrícola Panamericana, Zamorano, Honduras. Correo electrónico mknorops@gmail.com

${ }^{3}$ Bióloga y consultora ambiental. Correo electrónico luciaisa2@gmail.com

${ }^{4}$ Dasónomo y consultor independiente. Correo electrónico bany_q@yahoo.com
} 
Mora et al.: Caracterización del Bosque Seco Tropical Remanente en el Valle de Agalta, Honduras

\section{Introducción}

En Honduras existen remanentes de bosque tropical seco en la parte superior del valle del Aguán en el departamento de Yoro, el valle de Agalta en el departamento de Olancho y en las elevaciones bajas y medias del departamento de Santa Bárbara. Estos bosques tienen importancia ecológica y son el hábitat del colibrí esmeralda (Amazilia luciae), la única especie de ave endémica de Honduras y catalogada como en peligro de extinción (UICN 2011).

Los bosques del oriente (Valle del Aguán) son espinosos, de baja altura y con un dosel abierto, mientras que en el occidente (Santa Bárbara) son más húmedos, altos y con un dosel relativamente más cerrado (Anderson et al. 2010). Este bosque está compuesto por el arbustal deciduo, bosque deciduo bajo, bosque de galería (semideciduo) y el ecotono de pino-encino (INGTELSIG 2011). Estos bosques comparten características que los distinguen de otros ecosistemas, principalmente su estacionalidad muy marcada con épocas secas de hasta 7-8 meses de duración y bajas precipitaciones $(700 \mathrm{~mm} / \mathrm{año})$. Además, estos bosques se encuentran ubicados a sotavento de las principales cadenas montañosas (Stuart 1954) donde el calor y la sequedad del aire en la bajada de las laderas de las montañas han creado condiciones climáticas semiáridas (sombra de lluvia) que ha favorecido la formación de conjuntos únicos de especies con distribuciones muy restringidas.

Hace 50 años, la ecoregión semiárida en Honduras comprendía 1,957,284 ha, pero ha sido reducida drásticamente a 185,108 ha, 9.4\% del área original (Midence 2010). Los remanentes de este tipo de vegetación natural en buen estado de conservación están inmersos en un paisaje compuesto por una matriz de campos agrícolas y potreros de tenencia privada. No obstante, en el valle de Agalta la municipalidad de San Esteban ha protegido un bosque ejidal donde se ubica el botadero municipal. Este bosque es parte del fragmento más grande y continuo de Bosque Seco Tropical en todo el valle de Agalta.

La continua pérdida de bosque por tala y la expansión de la frontera agrícola, el sobrepastoreo y la pavimentación de la carretera entre Gualaco y San Esteban amenazan la supervivencia del Bosque Seco Tropical del valle de Agalta. Con su pérdida ha disminuido la posibilidad de conservación de especies de plantas endémicas y del colibrí esmeralda en el sitio, una especie que podría aportar recursos a la zona con el ecoturismo (Espinal y Mora 2012).

Este trabajo tiene como objetivo definir la distribución, diversidad y estructura del Bosque Seco Tropical del valle de Agalta. Es primordial identificar las especies de flora de importancia para la fauna, en particular para el colibrí esmeralda. Además consideramos de alto valor el determinar el área remanente, la estructura de los parches del ecosistema en cuestión, así como la condición de la tenencia de la tierra de estos parches. Toda esta información es importante para ayudar a entender la ecología del bosque seco, sus especies, incluido el colibrí esmeralda, y las posibles estrategias para la conservación de este ecosistema.

\section{Materiales y Métodos}

Área de estudio. El valle de Agalta tiene 55,308 ha y está ubicado entre los municipios de Gualaco y San Esteban, departamento de Olancho, Honduras. El terreno es $44.57 \%$ plano, $47.64 \%$ ligeramente ondulado y $6.57 \%$ muy ondulado. El valle se encuentra bordeado al norte por la montaña de Botaderos, al sur está la sierra de Agalta, al oeste la montaña de Jacaleapa y al este la sierra del río Tinto, que sirven de barrera natural para la humedad proveniente del Atlántico, lo que provoca la extrema condición seca del valle. Geográficamente, los límites del área de estudio están comprendidos entre las coordenadas 1660000 y 1692000 , al sur y norte, y 594000 y 642000 al oeste y este, respectivamente, en el datum WGS84 (Figura 1).

En el valle de Agalta existen varias series de suelos como los de valles Tomalá, Sulaco y Danlí. El clima en el valle pertenece a las siguientes provincias climáticas. Poco Lluvioso, con Invierno Seco (Yx) que se localiza en la comunidad de Gualaco, sus meses más lluviosos son octubre y noviembre mientras que los meses más secos son febrero y marzo. Lluvioso de Altura ( $\mathrm{Vx}$ ), comprende el valle de Gualaco y la Sierra de Agalta, sus meses más lluviosos son junio y septiembre con febrero y marzo como los más secos. Muy Lluvioso de Barlovento (Ek), se localiza en San Esteban, sus meses más lluviosos son julio y septiembre y los más secos marzo y abril. En promedio se registran $1,081 \mathrm{~mm}$ de lluvia al año y la humedad relativa promedio es de 82\% (Zúniga 1999). 


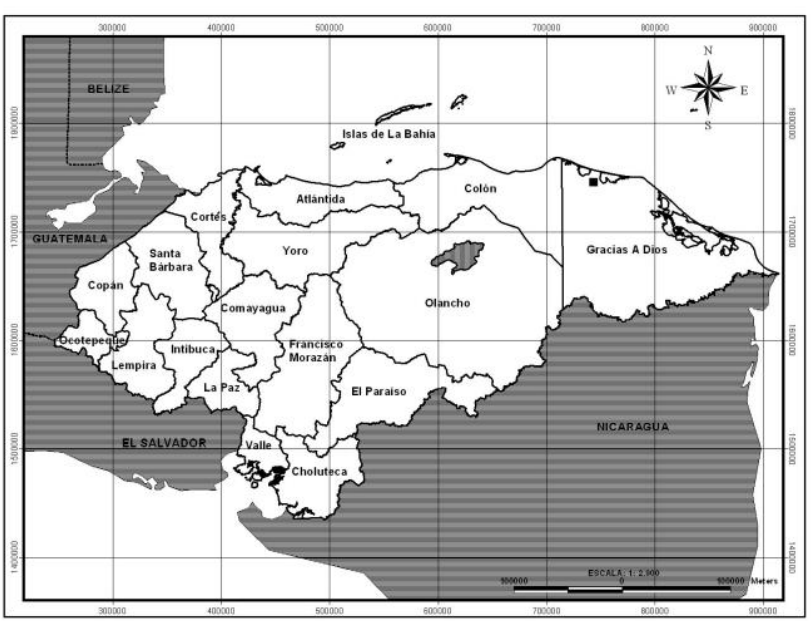

Figura 1. Valle de Agalta, en gris dentro del departamento de Olancho, Honduras.

Trabajo de campo. En agosto y septiembre de 2012 trabajamos en el valle de Agalta para identificar y caracterizar los remanentes de Bosque Seco Tropical según la clasificación del sistema de Holdridge (Holdridge 1962). Durante las giras de campo y con ayuda de las ortofotos identificamos y delimitamos los remanentes de bosque que estuvieran bien definidos y que conservaran vegetación natural relativamente bien conservada.

Para el inventario utilizamos un diseño de muestreo sistemático estratificado, con recorridos de senderos lineales en los parches de bosque. Establecimos 40 parcelas circulares según la apariencia y el grado de intervención sufrido por el parche. Las parcelas eran de $500 \mathrm{~m}^{2}$ (1/20 ha), con un radio de $12.62 \mathrm{~m}$. En cada una de ellas recopilamos información sobre la vegetación existente en formularios previamente elaborados. Clasificamos las especies por estrato (dosel, intermedio e inferior), medimos los diámetros a la altura del pecho (DAP) y estimamos las alturas de todos los árboles mayores de $5 \mathrm{~cm}$ de DAP.

Durante el levantamiento de la información identificamos las especies vegetales en el campo o recolectamos muestras para identificarlas posteriormente en el herbario de la UNAH
(Universidad Nacional Autónoma de Honduras). Para todas las especies anotamos su nombre común, provisto por informantes locales, para posteriormente obtener el nombre científico de los listados existentes para la flora de Honduras, particularmente de Nelson (2008).

Con los datos de campo calculamos la composición florística, la estructura del bosque y el Índice de Valor de Importancia de las especies (IVI) para cada asociación vegetal identificada. La composición florística la determinamos mediante el análisis de los géneros, especies y número de individuos por especie en cada asociación vegetal. La estructura vertical y horizontal la obtuvimos al correlacionar la distribución de los individuos y las especies con la altura, el diámetro y el área basal. El IVI de cada especie lo estimamos con las siguientes fórmulas:

Área basal $=3.1416 / 4 \times \mathrm{DAP}^{2}$

Abundancia relativa $=$ Número de individuos de una especie/Total de individuos de la muestra $\times 100$

Dominancia relativa = Área basal de una especie/ Área basal de todas las especies $\times 100$

$\mathbf{I V I}$ = área basal + abundancia relativa + dominancia relativa

\section{Resultados y Discusión}

En el valle de Agalta identificamos 20 parches de Bosque Seco Tropical con vegetación al menos similar a la original (Figura 2). Éstos tienen una cobertura vegetal formada por árboles (o rebrotes) de densidad mayor que la de las áreas circundantes, generalmente potreros. Los parches pueden proveer recursos de refugio y alimento a la fauna del lugar, incluido el colibrí esmeralda. Algunos guamiles o áreas de regeneración secundaria en estado avanzado, los consideramos parte de los parches boscosos debido a que cumplían las condiciones predefinidas. Estas áreas en regeneración, por lo general, contienen gran cantidad de recursos, particularmente frutos, semillas, néctar e insectos de gran importancia para la fauna (Blake y Loiselle 2001). 


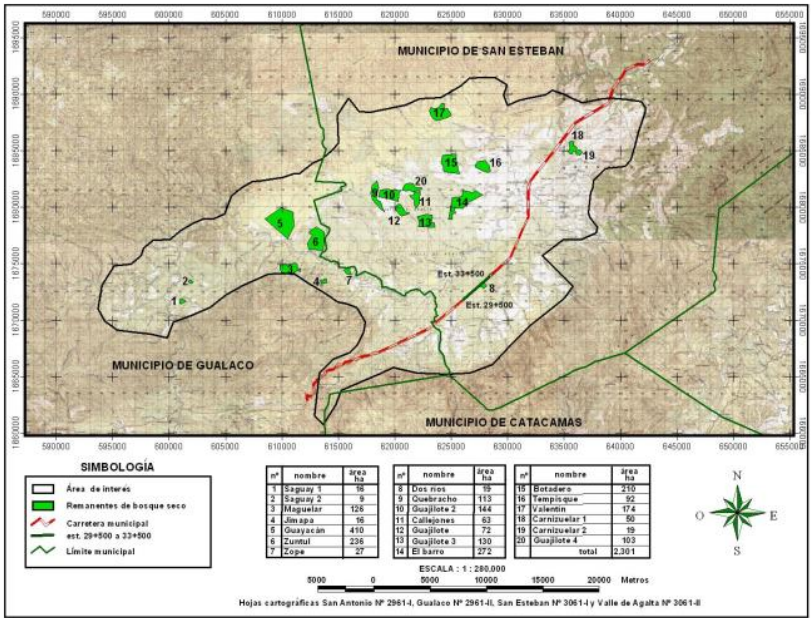

Figura 2. Remanentes o parches de Bosque Seco Tropical identificados y delimitados en el valle de Agalta, Olancho, Honduras, 2012.

Contabilizamos 265 especies de plantas, distribuidas en 78 familias y 223 géneros (Cuadro 1) en los fragmentos de bosque seco del valle de Agalta. Este número incluye algunas especies domésticas y otras introducidas. Las hierbas fueron el hábito dominante en el estudio con 81 especies, seguido de los árboles con 69 especies. Las familias más comunes fueron las leguminosas (Fabaceae) con 36 especies, las orquídeas con 17 especies y las asteráceas con 13 especies. Las especies más comunes y que tipifican al bosque seco del valle de Agalta son: biscuite (Acacia deamii), guamuche (Pithecellobium dulce), indio desnudo (Bursera simaruba), fierrillo (Matayba oppositifolia), Eugenia lempana, Eugenia coyolensis y Leucaena spp., así como el palanco (Luehea seemanii) y chaparro liso (Ehretia tinifolia) (Cuadro 1). En el sotobosque encontramos comúnmente especies de solanáceas como el frutico (Solanum diaboli) y el chichipate (Solanum hirtum). En este estrato dominaron especies propias de zonas en regeneración y las cactáceas, entre las que destacan la barba de viejo (Pilosocereus chrysacanthus), la pitahaya (Hylocereus undatus) y las oreja de vaca (Opuntia spp., al menos dos especies) que son abundantes.

Detectamos algunas especies de plantas endémicas de Honduras como el teocinte, Dioon mejiae (SERNA 2008). Esta especie se encuentra en la parte oeste del valle, en la comunidad de El
Saguay, donde observamos una alta concentración de individuos de esta especie (Figura 3). Esta planta es de gran utilidad ya que es comestible (Reyes y Suazo 2007). Sin embargo, su mayor valor está posiblemente en su potencial ecoturístico (Espinal y Mora 2012). También detectamos el guayabo de cerro (Eugenia lempana), el mestizo (Eugenia coyolensis) y la hierba Croton pendens. Estas especies endémicas de Honduras son elementos característicos del Bosque Seco Tropical e indicativas de las características biofísicas particulares de la conformación de este tipo de bosque (Holdridge 1962).

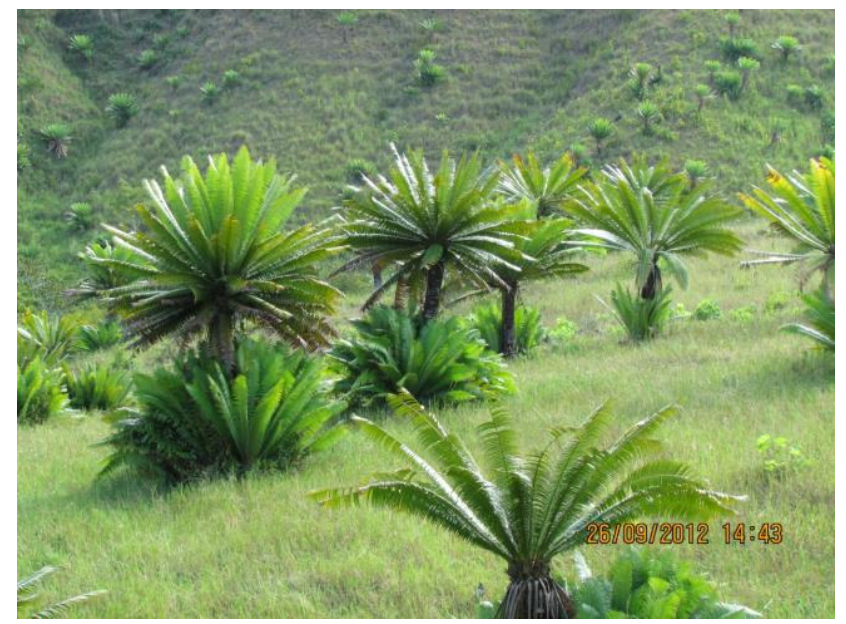

Figura 3. Plantas de teocinte (Dioon mejiae) en la comunidad del Saguay, Olancho, Honduras. Fotografía de Mario Espinal tomada el 26 de septiembre de 2012.

Desafortunadamente, el Bosque Seco Tropical es uno de los tipos de vegetación menos representado en las áreas protegidas de Honduras, a pesar de sus endemismos y la biodiversidad en general que contiene (Vreugdenhil et al. 2002). En general, este ecosistema es al que menos atención se le ha dado en Honduras aunque no solo protege un conjunto de especies vegetales características sino varias especies únicas en el mundo. Además, es el hábitat más utilizado por el colibrí esmeralda hondureño aun y cuando ya se sabe que su distribución es más amplia de lo que se había pensado (Germer 2012). 
Cuadro 1. Especies vegetales y hábito respectivo, encontradas en los remanentes de Bosque Seco Tropical y alrededores en el valle de Agalta, Olancho, Honduras. Incluye las especies domésticas e introducidas detectadas durante el trabajo de campo.

\begin{tabular}{|c|c|c|c|}
\hline Familia & Nombre Científico & Nombre Común & Hábito \\
\hline Acanthaceae & Sanchezia parvibracteata & Lotería & Arbusto \\
\hline Agavaceae & Furcraea cabuya & Pita & Hierba \\
\hline Agavaceae & Agave americana & Agave & Hierba \\
\hline Agavaceae & Yucca guatemalensis & Flor de izote & Hierba \\
\hline Amaranthaceae & Irisine diffusa & Mano de lagarto & Hierba \\
\hline Amaranthaceae & Amaranthus spinosus & Bledo & Hierba \\
\hline Anacardiaceae & Mangifera indica & Mango & Árbol \\
\hline Anacardiaceae & Spondias mombin & Jocote/jobo & Árbol \\
\hline Annonaceae & Annona sp. & Anona & Árbol \\
\hline Apiaceae & Sanicula liberta & Arracachillo & Hierba \\
\hline Apocynaceae & Echites yucatanensis & & Liana \\
\hline Apocynaceae & Mandevilla subsagittata & Flor de mico & Hierba trepadora \\
\hline Apocynaceae & Rauvolfia tetraphylla & Cola leche & Arbusto \\
\hline Apocynaceae & Tabernamontana amygdalifolia & Chilindrón & Árbol \\
\hline Apocynaceae & Tabernamontana alba & Cojón & Arbusto \\
\hline Apocynaceae & Catharanthus roseus & Guajaca & Hierba \\
\hline Apocynaceae & Plumeria alba & Flor de mayo & Árbol \\
\hline Araceae & Anthurium schlechtendalii & Gallinazo & Hierba \\
\hline Araceae & Anthurium sp. & Corazón & Hierba \\
\hline Araceae & Colocasia esculenta & Malanga & Hierba \\
\hline Araceae & Philodendron hederaceum & Caña de Cristo & Hierba \\
\hline Araceae & Philodendron angustisectum & Mano de león & Hierba \\
\hline Araceae & Syngonium podophyllum & Amor de hombre & Hierba \\
\hline Araceae & Zantedeschia aethiopica & Cartucho & Hierba \\
\hline Arecaceae & Acrocomia mexicana & Coyol & Palma \\
\hline Arecaceae & Attalea cohune & Corozo & Palma \\
\hline Arecaceae & Brahea dulcis & Suyate & Palma \\
\hline Arecaceae & Cocos nucifera & Coco & Palma \\
\hline Arecaceae & Dypsis lutescens & Palma areca & Palma \\
\hline Arecaceae & Roystonea regia & Cabiche & Palma \\
\hline Aristolochiaceae & Aristolochia anguicida & Canastilla & Bejuco \\
\hline Aristolochiaceae & Aristolochia maxima & Guaco/Canastilla & Bejuco \\
\hline Asclepiadaceae & Asclepias curassavica & Viborana & Hierba \\
\hline Asclepiadaceae & Calotropis procera & Huevo de gringo & Arbusto \\
\hline
\end{tabular}


Mora et al.: Caracterización del Bosque Seco Tropical Remanente en el Valle de Agalta, Honduras

Cuadro 1. Continuación.

\begin{tabular}{|c|c|c|c|}
\hline Familia & Nombre Científico & Nombre Común & Hábito \\
\hline Asteraceae & Ageratum conyzoides & Cola de alacrán & Hierba \\
\hline Asteraceae & Cirsium mexicanum & Cardo & Hierba \\
\hline Asteraceae & Cosmos sp. & Cambray & Hierba \\
\hline Asteraceae & Desmanthodium guatemalense & & Hierba \\
\hline Asteraceae & Emilia sonchifolia & Ambolia & Hierba \\
\hline Asteraceae & Perymenium ghiesbreghtii & Con & Arbusto \\
\hline Asteraceae & Stevia ovata & Flor de octubre & Hierba \\
\hline Asteraceae & Taraxacum officinale & Diente de león & Hierba \\
\hline Asteraceae & Telanthophora grandifolia & Tapatamal & Hierba \\
\hline Asteraceae & Tithonia rotundifolia & Girasol & Hierba \\
\hline Asteraceae & Verbesina turbacensis & Cube & Hierba \\
\hline Asteraceae & Zinnia peruviana & Ambolia & Hierba \\
\hline Bignoniaceae & Arrabidaea candicans & Matasanillo & Hierba \\
\hline Bignoniaceae & Cydista diversifolia & Bejuco de corral & Hierba trepadora \\
\hline Bignoniaceae & Crescentia alata & Cumba & Árbol \\
\hline Bignoniaceae & Macfadyena unguis-cati & Uña de gato & Hierba \\
\hline Bignoniaceae & Pyrostegia venusta & Flor de fuego & Hierba trepadora \\
\hline Bignoniaceae & Spathodea campanulata & Llama del bosque & Árbol \\
\hline Bignoniaceae & Tabebuia chrysantha & Cortez & Árbol \\
\hline Bignoniaceae & Tabebuia rosea & Macuelizo & Árbol \\
\hline Bixaceae & Bixa orellana & Achiote & Árbol \\
\hline Blechnaceae & Blechnum serrulatum & Petatillo & Helecho \\
\hline Bombacaceaea & Ceiba pentandra & Ceiba & Árbol \\
\hline Boraginaceae & Tournefortia volubilis & Comida de culebra & Hierba \\
\hline Boraginaceae & Cordia dentata & Tigüilote & Árbol \\
\hline Boraginaceae & Heliotropium indicum & Cola de alacrán & Hierba \\
\hline Bromeliaceae & Aechmea bracteata & Gallito & Hierba \\
\hline Bromeliaceae & Bromelia pinguin & Gallito & Hierba \\
\hline Bromeliaceae & Catopsis hahnii & Gallito & Hierba \\
\hline Bromeliaceae & Tillandsia fasciculata & Gallito & Hierba \\
\hline Bromeliaceae & Tillandsia balbisiana & Gallito & Hierba \\
\hline Bromeliaceae & Tillandsia schlechtendalii & Piñuela & Hierba \\
\hline Burseraceae & Bursera simaruba & Indio desnudo & Árbol \\
\hline Cactaceae & Acanthocereus tetragonus & Pitahaya & Arbusto \\
\hline Cactaceae & Hylocereus undatus & Hermosa & Bejuco \\
\hline Cactaceae & Nopalea guatemalensis & Oreja de vaca & Arbusto \\
\hline
\end{tabular}


Cuadro 1. Continuación.

\begin{tabular}{|c|c|c|c|}
\hline Familia & Nombre Científico & Nombre Común & Hábito \\
\hline Cactaceae & Opuntia guatemalensis & Oreja de vaca & Arbusto \\
\hline Cactaceae & Opuntia hondurensis & Oreja de vaca & Arbusto \\
\hline Cactaceae & Peniocereus hirschtianus & Cola de coyote & Epifita \\
\hline Cactaceae & Pilosocereus chrysacanthus & Barba de viejo & Arbusto \\
\hline Cactaceae & Rhipsalis baccifera & Quebradura & Epifita \\
\hline Campanulaceae & Lobelia laxiflora & Caballito & Hierba \\
\hline Capparaceae & Cratavea tapia & Naranjillo & Árbol \\
\hline Capparidaceae & Capparis cynophallophora & Azaharillo & Árbol \\
\hline Capparidaceae & Capparis indica & Carne de venado & Árbol \\
\hline Capparidaceae & Cleome spinosa & Ajonjolí de monte & Hierba \\
\hline Caricaceae & Carica papaya & Papaya & Arbusto \\
\hline Cecropiaceae & Cecropia peltata & Guarumo & Árbol \\
\hline Celestraceae & Crossopetalum parviflorum & Comida de pava & Hierba \\
\hline Chenopodiaceae & Chenopodium ambrosiodes & Apazote & Hierba \\
\hline Cladoniaceae & Cladonia spinosa & & Liquen \\
\hline Clusiaceae & Clusia sp. & Matapalo & Arbusto \\
\hline Cochlospermaceae & Cochlospermum vitifolium & Bombón & Arbusto \\
\hline Combretaceae & Combretum fruticosum & Peine de mico & Arbusto \\
\hline Combretaceae & Terminalia catappa & Almendro & Árbol \\
\hline Commelinaceae & Commelina diffusa & Diamela & Hierba \\
\hline Cactaceae & Opuntia guatemalensis & Oreja de vaca & Arbusto \\
\hline Cactaceae & Opuntia hondurensis & Oreja de vaca & Arbusto \\
\hline Cactaceae & Peniocereus hirschtianus & Cola de coyote & Epifita \\
\hline Cactaceae & Pilosocereus chrysacanthus & Barba de viejo & Arbusto \\
\hline Cactaceae & Rhipsalis baccifera & Quebradura & Epifita \\
\hline Campanulaceae & Lobelia laxiflora & Caballito & Hierba \\
\hline Capparaceae & Cratavea tapia & Naranjillo & Árbol \\
\hline Capparidaceae & Capparis cynophallophora & Azaharillo & Árbol \\
\hline Capparidaceae & Capparis indica & Carne de venado & Árbol \\
\hline Capparidaceae & Cleome spinosa & Ajonjolí de monte & Hierba \\
\hline Caricaceae & Carica papaya & Papaya & Arbusto \\
\hline Cecropiaceae & Cecropia peltata & Guarumo & Árbol \\
\hline Celestraceae & Crossopetalum parviflorum & Comida de pava & Hierba \\
\hline Chenopodiaceae & Chenopodium ambrosiodes & Apazote & Hierba \\
\hline Cladoniaceae & Cladonia spinosa & & Liquen \\
\hline Clusiaceae & Clusia sp. & Matapalo & Arbusto \\
\hline
\end{tabular}


Mora et al.: Caracterización del Bosque Seco Tropical Remanente en el Valle de Agalta, Honduras

Cuadro 1. Continuación.

\begin{tabular}{|c|c|c|c|}
\hline Familia & Nombre Científico & Nombre Común & Hábito \\
\hline Cochlospermaceae & Cochlospermum vitifolium & Bombón & Arbusto \\
\hline Combretaceae & Combretum fruticosum & Peine de mico & Arbusto \\
\hline Combretaceae & Terminalia catappa & Almendro & Árbol \\
\hline Commelinaceae & Commelina diffusa & Diamela & Hierba \\
\hline Convolvulaceae & Evolvulus ovatus & & Hierba \\
\hline Cucurbitaceae & Momordica charantia & Calaica & Hierba \\
\hline Cyperaceae & Cyperus sp. & Pelito de macho & Hierba \\
\hline Cyperaceae & Eleocharis palustris & Junco & Hierba \\
\hline Cyperaceae & Rhynchospora nervosa & Cola de burro & Hierba \\
\hline Dilleniaceae & Curatella americana & Friega traste & Arbusto \\
\hline Eleocarpacea & Muntigia calabura & Capulín & Árbol \\
\hline Euphorbiaceae & Acalypha diversifolia & Cordoncillo & Arbusto \\
\hline Euphorbiaceae & Acalypha firmula & Pelillo & Arbusto \\
\hline Euphorbiaceae & Caperonia castaneifolia & Serenillo & Hierba \\
\hline Euphorbiaceae & Croton ciliatoglandulosus & Pela nariz & Hierba \\
\hline Euphorbiaceae & Croton guatemalensis & Pela nariz & Hierba \\
\hline Euphorbiaceae & Croton pendens & & Hierba \\
\hline Euphorbiaceae & Cnidoscolus urens & Pica pica & Hierba \\
\hline Euphorbiaceae & Jatropha curca & Piñón & Arbusto \\
\hline Euphorbiaceae & Jatropha gossipiifolia & Piñón & Arbusto \\
\hline Euphorbiaceae & Pedilanthus camporum & Pie de niño & Hierba \\
\hline Euphorbiaceae & Ricinus communis & Higuera & Arbusto \\
\hline Fabaceae & Acacia collisii & Cachito & Arbusto \\
\hline Fabaceae & Acacia cookii & Cachito & Arbusto \\
\hline Fabaceae & Acacia ripari & Jamacuao & Árbol \\
\hline Fabaceae & Acacia farnesiana & Espino blanco & Arbusto \\
\hline Fabaceae & Acacia tenuifolia & Espino negro & Árbol \\
\hline Fabaceae & Andira inermis & Almendro de río & Árbol \\
\hline Fabaceae & Bauhinia divaricata & Casco de vaca & Arbusto \\
\hline Fabaceae & Bahuinia glabra & Escalera de mico & Bejuco trepador \\
\hline Fabaceae & Bauhinia variegata & Pata de vaca & Árbol \\
\hline Fabaceae & Caesalpinia pulcherrima & Barbona & Arbusto \\
\hline Fabaceae & Calliandra houstoniana & Barba de viejo & Arbusto \\
\hline Fabaceae & Canavalia villosa & Bejuco de gallina & Hierba \\
\hline Fabaceae & Cassia gradis & Carao & Árbol \\
\hline Fabaceae & Crotalaria retusa & Chinchín & Hierba \\
\hline
\end{tabular}


Cuadro 1. Continuación.

\begin{tabular}{|c|c|c|c|}
\hline Familia & Nombre Científico & Nombre Común & Hábito \\
\hline Fabaceae & Dalbergia glabra & Tinto & Arbusto \\
\hline Fabaceae & Delonix regia & Acacia roja & Árbol \\
\hline Fabaceae & Desmodium sericophyllum & Engordacabras & Hierba \\
\hline Fabaceae & Diphysa spinosa & Frijolillo & Hierba \\
\hline Fabaceae & Enterolobium cyclocarpum & Guanacaste & Árbol \\
\hline Fabaceae & Gliricidia sepium & Madreado & Árbol \\
\hline Fabaceae & Haemaeoxilum brasiletto & Brasileto & Arbusto \\
\hline Fabaceae & Inga vera & Guamo & Árbol \\
\hline Fabaceae & Leucaena sp. & Quebracho & Árbol \\
\hline Fabaceae & Lonchocarpus minimiflorum & Chaperno & Árbol \\
\hline Fabaceae & Lysiloma auritum & Quebracho & Árbol \\
\hline Fabaceae & Mimosa albida & Zarza & Arbusto \\
\hline Fabaceae & Mimosa pellita & Carbón & Hierba \\
\hline Fabaceae & Mimosa pudica & Dormilona & Hierba \\
\hline Fabaceae & Phaseolus lunatus & Frijol & Hierba \\
\hline Fabaceae & Pithecellobium dulce & Mangollano & Arbusto \\
\hline Fabaceae & Racosperma mangium & Acacia & Árbol \\
\hline Fabaceae & Samanea saman & Carreto & Árbol \\
\hline Fabaceae & Senna alata & Acacia amarilla & Arbusto \\
\hline Fabaceae & Senna reticulata & Baraja & Arbusto \\
\hline Fabaceae & Vatairea lundellii & Cucaracho & Árbol \\
\hline Fabaceae & Stizolobium pruriens & Pica pica & Hierba trepadora \\
\hline Flacourtiaceae & Casearia sp. & Espino & Árbol \\
\hline Flacurtiaceae & Xilosma flexuosum & Aguja de ara & Árbol \\
\hline Flacurtiaceae & Casearia corymbosa & Huevillo & Árbol \\
\hline Hydrophyllaceae & Wigandia urens & Chichicastón & Hierba \\
\hline Hypericaceae & Hypericum gnidioides & & Hierba \\
\hline Lamiaceae & Leonotis nepetifolia & Molinillo & Hierba \\
\hline Lamiaceae & Hyptis suaveolens & Chan & Hierba \\
\hline Lamiaceae & Vitex gaumeri & Cenizo azul & Árbol \\
\hline Lauraceae & Persea americana & Aguacate & Árbol \\
\hline Loganiaceae & Buddleia americana & Hoja blanca & Arbusto \\
\hline Loranthaceae & Phoradendron quadrangulare & Mata palo & Hemiparásita \\
\hline Loranthaceae & Psittacanthus schiedeanus & Muérdago & Hemiparásita \\
\hline Loranthaceae & Psittacanthus sp. & Suelda con suelda & Hemiparásita \\
\hline Loranthaceae & Struthanthus orbicularis & Tiricia & Hemiparásita \\
\hline
\end{tabular}


Mora et al.: Caracterización del Bosque Seco Tropical Remanente en el Valle de Agalta, Honduras

Cuadro 1. Continuación.

\begin{tabular}{|c|c|c|c|}
\hline Familia & Nombre Científico & Nombre Común & Hábito \\
\hline Lythraceae & Lagerstroemia speciosa & Flor de la reina & Arbusto \\
\hline Malpighiaceae & Malpighia glabra & Gueguecho & Árbol \\
\hline Malpighiaceae & Stigmaphyllon lindenianum & Mariposita & Arbusto \\
\hline Malpighiaceae & Byrsonima crassifolia & Nance & Árbol \\
\hline Malvaceae & Guazuma ulmifolia & Tapa culo & Árbol \\
\hline Malvaceae & Hibiscus rosa-sinensis & Mar pacífico & Arbusto \\
\hline Malvaceae & Luehea candida & Canastilla & Árbol \\
\hline Malvaceae & Luehea speciosa & Palanco & Árbol \\
\hline Malvaceae & Sida acuta & Escoba & Hierba \\
\hline Malvaceae & Wissadula hernandioides & Ojo de poeta & Hierba \\
\hline Melastomaceae & Miconia albicans & Sirin & Arbusto \\
\hline Melastomaceae & Miconia glaberrima & Sirin & Arbusto \\
\hline Melastomaceae & Miconia mexicana & Sirin & Arbusto \\
\hline Meliaceae & Cedrela odorata & Cedro & Árbol \\
\hline Meliaceae & Swietenia humilis & Caoba & Árbol \\
\hline Meliaceae & Trichilia sp. & Jocotillo & Arbusto \\
\hline Moraceae & Ficus maxima & Higuero & Árbol \\
\hline Myristicaceae & Virola guatemalensis & Sangre & Árbol \\
\hline Myrtaceae & Calyptranthes hondurensis & Arrayán & Árbol \\
\hline Myrtaceae & Eucalyptus globulus & Eucalipto común & Árbol \\
\hline Myrtaceae & Eugenia lempana EE & Guayabo de cerro & Árbol \\
\hline Myrtaceae & Eugenia coyolensis EE & Mestizo & Arbusto \\
\hline Myrtaceae & Psidium guayajava & Guayaba & Árbol \\
\hline Myrtaceae & Psidium salutare & Guayabilla & Árbol \\
\hline Nyctaginaceae & Neea psychotrioides & Cerezo & Árbol \\
\hline Nyctaginaceae & Guapira costaricana & Llanto & Árbol \\
\hline Onagraceae & Ludwigia octovalis & Clavillo & Hierba \\
\hline Onagraceae & Ludwigia sp. & Clavillo & Hierba \\
\hline Opiliaceae & Agonandra sp. & Naranjillo & Árbol \\
\hline Orchidaceae & Brassavola cucullata & Barba de bagre & Epifita \\
\hline Orchidaceae & Brassavola nodosa & Dama de la noche & Epifita \\
\hline Orchidaceae & Catasetum maculatum & Cebolleta & Epifita \\
\hline Orchidaceae & Dichaea glauca & Guaria morada & Epifita \\
\hline Orchidaceae & Encyclia nematocaulon & Cebollina & Epifita \\
\hline Orchidaceae & Epidendrum sp. & Orquídea & Epifita \\
\hline Orchidaceae & Guarianthe skinneri & Candelaria & Epifita \\
\hline
\end{tabular}


Cuadro 1. Continuación.

\begin{tabular}{|c|c|c|c|}
\hline Familia & Nombre Científico & Nombre Común & Hábito \\
\hline Orchidaceae & Laelia rubescens & Flor de Jesús & Epifita \\
\hline Orchidaceae & Myrmecophilla tibicinis & Caño & Epifita \\
\hline Orchidaceae & Oncidium carthagenense & San Andrés & Epifita \\
\hline Orchidaceae & Oncidium sp. & Orquídea & Epifita \\
\hline Orchidaceae & Prosthechea sp. & Orquídea & Epifita \\
\hline Orchidaceae & Prosthechea fragrans & Lirio & Epifita \\
\hline Orchidaceae & Schomburgkia superbiens & Candelaria & Epifita \\
\hline Orchidaceae & Trichocentrum cebolleta & Cebolla de jícaro & Epifita \\
\hline Orchidaceae & Trigonidium egertonianum & Boca de dragón & Epifita \\
\hline Orchidaceae & Vanilla planifolia & Vainilla & Hierba trepadora \\
\hline Oxalidaceae & Oxalis sp. & Trébol & Hierba \\
\hline Petiveriaceae & Petiveria alliacea & Ajillo & Hierba \\
\hline Phytolaccaceae & Phytolacca rivinoides & Jaboncillo & Hierba \\
\hline Phytolaccaceae & Rivina humilis & Coralillo & Hierba \\
\hline Pinaceae & Pinus oocarpa & Pino ocote & Árbol \\
\hline Pinaceae & Pinus caribaea var. hondurensis & Pino costero & Árbol \\
\hline Piperaceae & Peperomia acuminata & Soledad & Hierba \\
\hline Piperaceae & Piper aduncum & Cordoncillo & Arbusto \\
\hline Piperaceae & Piper auritum & Cordoncillo & Arbusto \\
\hline Piperaceae & Piper jacquemontianum & Cordoncillo & Arbusto \\
\hline Poaceae & Andropogon bicornis & Cola de burro & Hierba \\
\hline Poaceae & Chloris rufescens & Zacate estrella & Hierba \\
\hline Poaceae & Hyparrhenia rufa & Jaragua & Hierba \\
\hline Poaceae & Lasiacis procerrima & Carricillo & Hierba \\
\hline Poaceae & Melinis repens & Flor moradita & Hierba \\
\hline Poaceae & Oplismenus sp. & Pasto & Hierba \\
\hline Poaceae & Megathyrsus maximus & Guinea & Hierba \\
\hline Poaceae & Paspalum botterii & Pasto & Hierba \\
\hline Poaceae & Pennisetum sp. & Pasto elefante & Hierba \\
\hline Poaceae & Setaria parviflora & Zacate sedoso & Hierba \\
\hline Poaceae & Sporobolus jacquemontii & Estrella & Hierba \\
\hline Poaceae & Zea mays & Maíz & Hierba \\
\hline Polipodeaceae & Phlebodium sp. & & Helecho \\
\hline Polygonaceae & Coccoloba acapulcensis & Tolondrón & Arbusto \\
\hline Polygonaceae & Polygonum hydropiperoides & Chile de perro & Hierba \\
\hline Polygonaceae & Podopterus mexicana & Escabrón & Árbol \\
\hline
\end{tabular}


Mora et al.: Caracterización del Bosque Seco Tropical Remanente en el Valle de Agalta, Honduras

Cuadro 1. Continuación.

\begin{tabular}{|c|c|c|c|}
\hline Familia & Nombre Científico & Nombre Común & Hábito \\
\hline Polygonaceae & Neomeollspaughia paniculata & Tapa tamal & Arbusto \\
\hline Pontederiaceae & Heteranthera sp. & & Hierba \\
\hline Portulacaceae & Talinum paniculatum & Rama de sapo & Hierba \\
\hline Portulacaceae & Portulacca pilosa & Amor de un rato & Hierba \\
\hline Primulaceae & Parathesis sp. & Uva & Arbusto \\
\hline Pteridaceae & Adiantum princeps & Canastilla & Helecho \\
\hline Pteridaceae & Pityrogramma calomelanos & Cola de pescado & Helecho \\
\hline Pteridaceae & Pteris grandifolia & Mano de león & Helecho \\
\hline Pteridaceae & Pteris longifolia & & Helecho \\
\hline Rhamnaceae & Ziziphus mauritiana & Yuyugo & Arbusto \\
\hline Rubiaceae & Genipa americana & Butunte & Arbusto \\
\hline Rubiaceae & Morinda citrifolia & Noni & Arbusto \\
\hline Rubiaceae & Randia armata & Cruserito & Arbusto \\
\hline Rutaceae & Amyris elmifera & Chilillo & Árbol \\
\hline Rutaceae & Megastigma skinneri & Culantrillo & Arbusto \\
\hline Salicaceae & Salix humboldtiana & Sauce & Árbol \\
\hline Sapindaceae & Matayba oppositifolia & Fierrillo & Arbusto \\
\hline Sapindaceae & Paullinia fuscescen & Bejuco de aparejo & Bejuco \\
\hline Sapindaceae & Thouinia decandrum & Pescadillo & Árbol \\
\hline Sapotaceae & Sideroxilon capiri & Tempisque & Hierba \\
\hline Sapotaceae & Sideroxylon obtusifolium & Cagalera & Árbol \\
\hline Sapotaceae & Sideroxylon stenospermum & & Árbol \\
\hline Simarubaceae & Simarouba glauca & Aceituno negro & Árbol \\
\hline Solanaceae & Capsicum annuum & Chile & Arbusto \\
\hline Solanaceae & Solanum americanum & Güistomate & Arbusto \\
\hline Solanaceae & Solanum capsioldes & Chichigua & Arbusto \\
\hline Solanaceae & Solanum hirtum & Arrayán & Arbusto \\
\hline Solanaceae & Solanum torvum & Frutico & Arbusto \\
\hline Sterculaceae & Melochia sp. & Escoba & Hierba \\
\hline Thelypteridaceae & Thelypteris hispidula & Canastilla & Helecho \\
\hline Theophrastaceae & Jacquinia nitida & Siempre viva & Árbol \\
\hline Ulmaceae & Trema micrantha & Capulín & Árbol \\
\hline Ulmaceae & Phyllostylon rhamnoides & Palo blanco & Árbol \\
\hline Verbenaceae & Lantana camara & Cinco negritos & Hierba \\
\hline Zamiaceae & Dioon mejiae EE & Teocinte & Árbol \\
\hline
\end{tabular}

$\mathrm{EE}=$ Especie endémica. 
Estructura de la vegetación. El bosque del valle de Agalta está entre los 200 y $600 \mathrm{msnm}$, tiene una precipitación que oscila entre 1,000 y $2,000 \mathrm{~mm}$ por año, temperaturas promedio de $24{ }^{\circ} \mathrm{C}$ y seis meses de estación seca, lo que lo ubica como Bosque Seco Tropical en la clasificación de zonas de vida de Holdridge (Holdridge 1962). Las áreas de vegetación natural contienen bosques latifoliados deciduos 0 semideciduos, desde semidensos a densos, con excepción de los parches de fagáceas (Fagaceae) que se encuentran en las partes altas de algunos cerros, a mayor altura con relación al nivel del valle. Estas diferencias en densidades y especies han sido propiciadas por las distintas conformaciones topográficas y edáficas que existen en el área, además del grado de intervención antrópica sufrida.

Ecosistemas. Es difícil identificar con precisión los ecosistemas existentes en el valle de Agalta, ya que se mezclan constantemente con predominancia de los sistemas productivos antropogénicos. En este último sistema se encuentran plantas que sugieren que hubo otro tipo de ecosistema. El indio desnudo (Bursera simaruba) y otras especies encontradas en las orillas de las quebradas o antiguos correderos de agua, como el cedro (Cedrela odorata) y la ceiba (Ceiba pentandra) sugieren la existencia en el pasado de ecosistemas más húmedos. Esto se debe principalmente a la poca extensión horizontal del bosque seco en estado natural, además de las alteraciones antropogénicas que se han dado y se dan en la zona. Se puede decir que casi todo el bosque bajo análisis es un sistema agropecuario con distintos grados de descanso debido a los bajos precios del ganado de carne. Aun así, de acuerdo a la clasificación de UNESCO, identificamos dos asociaciones vegetales en el área de estudio: el bosque deciduo bajo y el bosque de pino-encino, o ecotono de pino-encino, como ha sido calificado por algunos autores (INGTELSIG 2013).

Bosque deciduo bajo. Este sistema vegetal está caracterizado por una cobertura vegetal de especies deciduas y algunas siempreverdes que forman un dosel de entre 5 y $15 \mathrm{~m}$ de altura. En este bosque se encuentran especies emergentes de hasta $30 \mathrm{~m}$, cubren un sotobosque formado por arbustos y latizales de los árboles dominantes o superiores. El suelo está cubierto por hierbas en la época de lluvias y descubierto en la época seca, con especies como brasil (Haematoxyllum brasiletto), guayabillo (Eugenia lempana), tolondrón (Coccoloba acapulcensis). Se pueden encontrar algunas suculentas rastreras 0 arbóreas. Al igual, se encuentran algunas especies como el cortés (Tabebuia guayacan), jagua (Genipa americana), macuelizo (Tabebuia rosea) y laurel (Cordia alliodora) (INGTELSIG 2011).

En el valle de Agalta, el bosque deciduo bajo está representado por especies de mimosifolias (Mimosaceae) como el biscuite (Acacia deamii), el guamuche (Pithecellobium dulce), que aquí reemplaza al brasil (Haematoxyllum brasileto); indio desnudo (Bursera simaruba), fierrillo (Matayba oppositifolia), ejemplares de Eugenia lempana y E. coyolensis y leucaenas (Leucaena spp.). Además, encontramos por todo el sitio de estudio varios ejemplares de palanco (Luehea seemanii) y de chaparro liso (Ehretia tinifolia).

En el sotobosque se encuentran generalmente especies de solanáceas (Solanaceae) como el frutico (Solanum diaboli), chichipate (Solanum hirtum) y una gran cantidad de especies dominantes en la regeneración, además de especies de la familia Cactaceae (INGTELSIG 2011). Dentro de estas últimas encontramos barba de viejo (Pilosocereus chrysacanthus), pitahaya (Peniocereus chiapensis) y oreja de vaca (Opuntia spp.).

En este ecosistema se encuentran algunos tipos de zarza y bromeliáceas (Bromeliaceae) como la piñuela (Bromelia plumierı) y, según el tipo de suelo, se encuentran ciertas herbáceas como la malva negra (Sida acuta) y la comelina (Commelina diffusa) (INGTELSIG 2011). También existe trepadoras como el chupamiel (Combretum fruticosum), que sirve de alimento al colibrí esmeralda, la escalera de mico (Bahuinia glabra), especies de pelanaríz (Croton spp.) y tripa de pollo o comelina (Commelina diffusa) (INGTELSIG 2011). Encontramos también algunos arbustales microfoliados compuestos en su mayoría por biscuite (Acacia deamii), principalmente en áreas que formaron parte de propiedades agrícolas. Estos arbustales se han considerado como una etapa en la sucesión hacia la formación de un bosque deciduo bajo (INGTELSIG 2011).

Bosque de pino-encino. Este bosque forma una zona de transición (ecotono) entre el bosque deciduo bajo y el bosque de pino. Aunque algunas veces puede cubrir extensiones considerables de terreno, en este caso se restringe a franjas delgadas cubiertas 
Mora et al.: Caracterización del Bosque Seco Tropical Remanente en el Valle de Agalta, Honduras

por una mezcla de encinos (Quercus corrugata), nances (Byrsonima crassifolia), chaparros (Curatella americana) y quebrachos (Lysiloma spp.). Generalmente, es el ecosistema siguiente en ser destruido luego de que se ha arrasado con los bosques deciduos bajos.

Otros ecosistemas. En el área entre Gualaco y San Esteban encontramos otros ecosistemas que no describimos aquí ya que son artificiales o no tienen significancia directa para este estudio y sus objetivos. Éstos son el sistema productivo antropogénico (sistema agropecuario) que ocupa la mayoría del área de estudio (varios guamiles-regeneración secundaria, fueron incluidos como bosque deciduo bajo en el análisis); el sistema urbano, formado por los caseríos, poblados, carreteras y demás infraestructura urbana y el sistema acuático formado por los ríos Grande, Alao, Tonjagua, sus afluentes y una gran cantidad de pequeñas lagunas naturales y artificiales.

Estructura vertical. Analizamos por separado la composición vertical de los doseles debido a que los promedios de cada uno de los dos ecosistemas resultaron muy dispares. En el bosque deciduo bajo, los valores están concentrados en el dosel entre 5 y 7 $\mathrm{m}$ de altura con pocos individuos que sobresalen $\mathrm{y}$ pocos individuos establecidos debajo de esta categoría (Cuadro 1). La mayoría de los sitios inventariados están constituidos por bosques alterados con una gran cantidad de troncos con rebrotes. Igual ocurre en el bosque de pino-encino en el que el dosel llega solamente a 6 - $10 \mathrm{~m}$ de altura, sin árboles emergentes (Cuadro 2).

Estructura horizontal: abundancia. El biscuite (Acacia deamii) fue la especie con la abundancia relativa más alta, seguido por el guamuche (Pithecelobium dulce) (Cuadro 3). Lo anterior puede ser el resultado de los rebrotes de las plantas existentes del bosque original. Éstos poseen varios fustes en un solo pie, válidos dentro de los parámetros de diámetro mínimo considerado en el inventario.

En el bosque de pino-encino dominan las fagáceas. Las muestras fueron tomadas en un rodal aislado en una colina baja, con una mezcla de dos especies de Quercus y nance. Encontramos 17 individuos de encino (Quercus sapoetifolia), 14 de roble (Quercus segoviensis) ambas fagáceas y 10 de nance (Byrsonima crassifolia, Malpighiaceae).
Cuadro 2. Composición vertical por ecosistema en el valle de Agalta entre Gualaco y San Esteban, Olancho, Honduras.

\begin{tabular}{llc}
\hline Ecosistema & Estrato & $\begin{array}{c}\# \\
\text { Individuos }\end{array}$ \\
\hline \multirow{3}{*}{ Bosque deciduo bajo } & $>5 \mathrm{~m}$ & 36 \\
& Entre 5 y $7 \mathrm{~m}$ & 332 \\
& $>7 \mathrm{~m}$ & 41 \\
\hline \multirow{3}{*}{ Bosque roble-encino } & $<6 \mathrm{~m}$ & 15 \\
& Entre 6 y $10 \mathrm{~m}$ & 26 \\
& $>10 \mathrm{~m}$ & 0 \\
\hline
\end{tabular}

Dominancia. La dominancia, reflejada por la participación de cada especie en la conformación del área basal por hectárea, en el bosque deciduo bajo fue más alta para el guamuche (Pithecellobium dulce), seguido por el biscuite (Acacia deamii) (Cuadro 4). Estas dos especies tienen una alta dominancia debido a la acumulación del área basal de la gran cantidad de individuos muestreados. No encontramos diámetros grandes por ser casi todos bosques alterados; la frecuencia de individuos encontrados por especie marcó la pauta en la dominancia. Varias especies estuvieron representadas por una dominancia menor a cero (Cuadro 4).

El roble, debido a la mayor cantidad de individuos y a diámetros ligeramente mayores, domina en el bosque de pino-encino (Cuadro 5). Sin embargo, la participación de algunos individuos de nance con diámetros mayores al resto de especies lo lleva a alcanzar el tercer lugar, aunque distante de los encinos (Cuadro 5).

Índice de Valor de Importancia. El Índice de Valor de Importancia (IVI) señala la importancia relativa de cada especie en una comunidad, de acuerdo a su dominancia y abundancia. En el valle de Agalta el biscuite y el guamuche son las especies con el mayor IVI (Cuadro 6). Este resultado refleja el estado de intervención antropogénica en el bosque, caracterizado por mayor cantidad de individuos de las especies que poseen mayor capacidad de rebrotes y más resistencia a los incendios en los sitios ganaderos. EI IVI del bosque de pino-encino está balanceado (Cuadro 7). Lo anterior, indica una participación dominante de las fagáceas. Sin embargo, el nance tiene su cuota de importancia. 
Cuadro 3. Abundancia relativa de cada especie de árbol del bosque deciduo bajo del valle de Agalta entre Gualaco y San Esteban, Olancho, Honduras.

\begin{tabular}{lllcc}
\hline Nombre Común & Nombre Científico & Familia & \# Árboles & Abundancia Relativa \\
\hline Biscuite & Acacia deamii & Mimosoideae & 148 & 36.19 \\
\hline Guamuche & Pithecelobium dulce & Mimosoideae & 124 & 30.32 \\
\hline Indio desnudo & Bursera simaruba & Burseraceae & 13 & 3.18 \\
\hline Tolondrón & Cocoloba acapulcensis & Polygonaceae & 21 & 5.13 \\
\hline Amarrajabón & Neomillspaughia paniculata & Polygonaceae & 25 & 6.11 \\
\hline Frijolillo & Leucaena sp. & Mimosoideae & 20 & 4.89 \\
\hline Palanco & Luehea candida & Malvaceae & 13 & 3.18 \\
\hline Azajarillo & Capparis admirabilis & Capparidaceae & 3 & 0.73 \\
\hline Fierrillo & Matayba oppositifolia & Myrtaceae & 12 & 2.93 \\
\hline Quebracho & Lysiloma sp. & Mimosoideae & 3 & 0.73 \\
\hline Bombón & Cochlospermum vitifolium & Cochlospermaceae & 5 & 1.22 \\
\hline Guayabillo & Eugenia lempana & Myrtaceae & 5 & 1.22 \\
\hline Jícaro & Crescentia alata & Bignoniaceae & 2 & 0.49 \\
\hline Tambor & Gyrocarpus americanus & Hernandiaceae & 2 & 0.49 \\
\hline Chaparro liso & Ehretia tinifolia & Boraginaceae & 2 & 0.49 \\
\hline Espino verde & Chloroleucon mangense & Mimosoideae & 2 & 0.49 \\
\hline Purga & Trichilia sp. & Meliaceae. & 1 & 0.24 \\
\hline Flor de mayo & Plumeria alba & Apocynaceae & 1 & 0.24 \\
\hline Arrayán & Calyptrantes hondurensis & Myrtaceae & 2 & 0.49 \\
\hline Huevo de gato & Thevetia ahouai & Apocynaceae & 1 & 0.24 \\
\hline Guayabo & Psidium guajava & Myrtaceae & 1 & 0.24 \\
\hline Palo amarillo & Phyllostylon rhamniodes & Ulmaceae & 1 & 0.24 \\
\hline Cachito & Acacia farnesiana & Mimosoideae & 1 & 0.24 \\
\hline Frutillo & Casearia coimbosa & Salicaceae & 1 & 0.24 \\
\hline & & & 409 & \\
\hline
\end{tabular}

Tenencia de la tierra. Los remanentes de bosques delimitados en el valle de Agalta (Figura 1) están conformados por 20 fragmentos de Bosque Seco Tropical, 19 son de tenencia privada y uno de tenencia municipal y privada (Cuadro 8). Los terrenos se encuentran dispersos a lo largo y ancho del valle, tiene una extensión de 2,301 ha. Nombramos los fragmentos según su ubicación geográfica y con referencia al nombre de localidades conocidas (Cuadro 7). De los 20 fragmentos de bosque seco, siete se encuentran en la jurisdicción del municipio de
Gualaco y 13 dentro del municipio de San Esteban.

La existencia de plantas endémicas y de distribución restringida $y$ de familias como cactáceas y bromeliáceas, subrayan la gran importancia biológica de los bosques secos hondureños (SERNA 2008). Pero la existencia de vías de acceso, el avance de la frontera agrícola y otros factores antropogénicos son determinantes en la fragmentación del Bosque Seco Tropical del valle de Agalta. Esto ha provocado la conversión del bosque seco a sistemas agrícolas, ganaderos y áreas urbanas (Quiel y Pineda 2010). 
Mora et al.: Caracterización del Bosque Seco Tropical Remanente en el Valle de Agalta, Honduras

Cuadro 4. Área basal y dominancia relativa por especie en el bosque deciduo bajo del valle de Agalta entre Gualaco y San Esteban, Olancho, Honduras.

\begin{tabular}{|c|c|c|c|}
\hline Nombre Común & Nombre Científico & $\begin{array}{c}\text { Área Basal } \\
\left(\mathrm{m}^{2} / \mathrm{ha}\right)\end{array}$ & Dominancia Relativa \\
\hline Biscuite & Acacia deamii & 1.25 & 23.95 \\
\hline Guamuche & Pithecelobium dulce & 1.50 & 28.81 \\
\hline Indio desnudo & Bursera simaruba & 0.65 & 12.43 \\
\hline Tolondrón & Cocoloba acapulcensis & 0.20 & 3.88 \\
\hline Amarrajabón & Neomillspaughia paniculata & 0.15 & 2.83 \\
\hline Frijolillo & Leucaena sp. & 0.17 & 3.24 \\
\hline Palanco & Luehea candida & 0.17 & 3.24 \\
\hline Azajarillo & Capparis admirabilis & 0.24 & 4.52 \\
\hline Fierrillo & Matayba oppositifolia & 0.08 & 1.59 \\
\hline Quebracho & Lysiloma sp. & 0.17 & 3.33 \\
\hline Bombón & Cochlospermum vitifolium & 0.13 & 2.45 \\
\hline Guayabillo & Eugenia lempana & 0.13 & 2.41 \\
\hline Jícaro & Crescentia alata & 0.14 & 2.70 \\
\hline Tambor & Gyrocarpus americanus & 0.08 & 1.48 \\
\hline Chaparro liso & Ehretia tinifolia & 0.07 & 1.36 \\
\hline Espino verde & Chloroleucon mangense & 0.02 & 0.42 \\
\hline Purga & Trichilia sp. & 0.02 & 0.45 \\
\hline Flor de mayo & Plumeria alba & 0.02 & 0.38 \\
\hline Arrayán & Calyptrantes hondurensis & 0.01 & 0.08 \\
\hline Huevo de gato & Thevetia ahouai & 0.01 & 0.13 \\
\hline Guayabo & Psidium guajava & 0.01 & 0.10 \\
\hline Palo amarillo & Phyllostylon rhamniodes & 0.01 & 0.08 \\
\hline Cachito & Acacia farnesiana & 0.01 & 0.08 \\
\hline Frutillo & Casearia coimbosa & 0.01 & 0.08 \\
\hline Total & & 5.00 & 100.00 \\
\hline
\end{tabular}

Cuadro 5. Área basal y dominancia relativa por especie en el bosque de pino-encino en el valle de Agalta entre Gualaco y San Esteban, Olancho, Honduras.

\begin{tabular}{llcc}
\hline Nombre Común & Nombre Científico & Área Basal $\left.\mathbf{~ ( ~}^{2} / \mathbf{h a}\right)$ & Dominancia Relativa \\
\hline Roble & Quercus sapoetifolia & 5.19 & 53.34 \\
\hline Encino & Quercus segoviensis & 3.38 & 34.67 \\
\hline Nance & Byrsonima crassifolia & 1.16 & 11.99 \\
\hline \multicolumn{1}{c}{ Total } & & 9.74 & 100.00 \\
\hline
\end{tabular}


Cuadro 6. Índice de Valor de Importancia (IVI) y parámetros asociados (incluidos $A R=$ abundancia relativa y $\mathrm{DR}=$ dominancia relativa) por especie en el bosque deciduo bajo en el valle de Agalta entre Gualaco y San Esteban, Olancho, Honduras.

\begin{tabular}{|c|c|c|c|c|c|c|c|c|c|c|}
\hline $\begin{array}{l}\text { Nombre } \\
\text { Común }\end{array}$ & $\begin{array}{l}\text { Nombre } \\
\text { Científico }\end{array}$ & Familia & $\begin{array}{c}\# \\
\text { Plantas }\end{array}$ & $\begin{array}{c}\text { Altura } \\
\text { (m) }\end{array}$ & $\begin{array}{c}\text { Árboles } \\
\text { /ha }\end{array}$ & $\begin{array}{l}\mathrm{DAP}^{\S} \\
(\mathrm{cm})\end{array}$ & $\begin{array}{c}\text { Área } \\
\text { Basal } \\
\left(\mathrm{m}^{2} / \mathrm{ha}\right)\end{array}$ & AR & DR & IVI \\
\hline Biscuite & Acacia deamii & Mimosoideae & 148 & 6 & 118.4 & 11.6 & 1.25 & 36.19 & 23.95 & 60.13 \\
\hline Guamuche & $\begin{array}{l}\text { Pithecelobium } \\
\text { dulcis }\end{array}$ & Mimosoideae & 124 & 6 & 99.2 & 13.9 & 1.50 & 30.32 & 28.81 & 59.13 \\
\hline Indio desnudo & $\begin{array}{l}\text { Bursera } \\
\text { simaruba }\end{array}$ & Burseraceae & 13 & 10 & 10.4 & 28.2 & 0.65 & 3.18 & 12.43 & 15.61 \\
\hline Tolondrón & $\begin{array}{l}\text { Cocoloba } \\
\text { acapulcensis }\end{array}$ & Polygonaceae & 21 & 5 & 16.8 & 12.4 & 0.20 & 5.13 & 3.88 & 9.02 \\
\hline Amarra jabón & $\begin{array}{l}\text { Neomillspaughia } \\
\text { paniculata }\end{array}$ & Polygonaceae & 25 & 6 & 20.0 & 9.7 & 0.15 & 6.11 & 2.83 & 8.94 \\
\hline Frijolillo & Leucaena sp. & Mimosoideae & 20 & 7 & 16.0 & 11.6 & 0.17 & 4.89 & 3.24 & 8.13 \\
\hline Palanco & Luehea candida & Malvaceae & 13 & 9 & 10.4 & 14.4 & 0.17 & 3.18 & 3.24 & 6.42 \\
\hline Azajarillo & $\begin{array}{l}\text { Capparis } \\
\text { admirabilis }\end{array}$ & Capparidaceae & 3 & 5 & 2.4 & 35.4 & 0.24 & 0.73 & 4.52 & 5.25 \\
\hline Fierrillo & $\begin{array}{l}\text { Matayba } \\
\text { oppositifolia }\end{array}$ & Myrtaceae & 12 & 7 & 9.6 & 10.5 & 0.08 & 2.93 & 1.59 & 4.52 \\
\hline Quebracho & Lysiloma sp. & Mimosoideae & 3 & 7 & 2.4 & 30.4 & 0.17 & 0.73 & 3.33 & 4.07 \\
\hline Bombón & $\begin{array}{l}\text { Cochlospermum } \\
\text { vitifolium }\end{array}$ & $\begin{array}{l}\text { Cochlo- } \\
\text { spermaceae }\end{array}$ & 5 & 8 & 4.0 & 20.2 & 0.13 & 1.22 & 2.45 & 3.68 \\
\hline Guayabillo & Eugenia lempana & Myrtaceae & 5 & 6 & 4.0 & 20.0 & 0.13 & 1.22 & 2.41 & 3.63 \\
\hline Jícaro & Crescentia alata & Bignoniaceae & 2 & 7 & 1.6 & 33.5 & 0.14 & 0.49 & 2.70 & 3.19 \\
\hline Tambor & $\begin{array}{l}\text { Gyrocarpus } \\
\text { americanus }\end{array}$ & Hernandiaceae & 2 & 9 & 1.6 & 24.8 & 0.08 & 0.49 & 1.48 & 1.97 \\
\hline Chaparro liso & Ehretia tinifolia & Boraginaceae & 2 & 8 & 1.6 & 23.8 & 0.07 & 0.49 & 1.36 & 1.85 \\
\hline Espino verde & $\begin{array}{l}\text { Chloroleucon } \\
\text { mangense }\end{array}$ & Mimosoideae & 2 & 6 & 1.6 & 13.2 & 0.02 & 0.49 & 0.42 & 0.91 \\
\hline Purga & Trichilia sp. & Meliaceae & 1 & 8 & 0.8 & 19.3 & 0.02 & 0.24 & 0.45 & 0.69 \\
\hline Flor de mayo & Plumeria alba & Apocynaceae & 1 & 7 & 0.8 & 17.7 & 0.02 & 0.24 & 0.38 & 0.62 \\
\hline Arrayán & $\begin{array}{l}\text { Calyptrantes } \\
\text { hondurensis }\end{array}$ & Myrtaceae & 2 & 6 & 1.6 & 5.9 & 0.01 & 0.49 & 0.08 & 0.57 \\
\hline Huevo de gato & Thevetia ahouai & Apocynaceae & 1 & 5 & 0.8 & 10.2 & 0.01 & 0.24 & 0.13 & 0.37 \\
\hline Guayabo & Psidium guajava & Myrtaceae & 1 & 6 & 0.8 & 9.1 & 0.01 & 0.24 & 0.10 & 0.34 \\
\hline Pasacaite & No identificada & & 1 & 4 & 0.8 & 7.9 & 0.01 & 0.24 & 0.08 & 0.32 \\
\hline Cachito & $\begin{array}{l}\text { Acacia } \\
\text { farnesiana }\end{array}$ & Mimosoideae & 1 & 4 & 0.8 & 7.9 & 0.01 & 0.24 & 0.08 & 0.32 \\
\hline Frutillo & $\begin{array}{l}\text { Casearia } \\
\text { coimbosa }\end{array}$ & Salicaceae & 1 & 5 & 0.8 & 7.9 & 0.01 & 0.24 & 0.08 & 0.32 \\
\hline Total & & & 409 & & 327.0 & & 5.00 & 100 & 100 & 200 \\
\hline
\end{tabular}

${ }^{\S}$ Diámetro a la altura del pecho. 
Mora et al.: Caracterización del Bosque Seco Tropical Remanente en el Valle de Agalta, Honduras

Cuadro 7. Índice de Valor de Importancia (IVI) y parámetros asociados (incluidos $A R=$ abundancia relativa y $\mathrm{DR}=$ dominancia relativa) por especie en el bosque de pino-encino en el valle de Agalta entre Gualaco y San Esteban, Olancho, Honduras.

\begin{tabular}{lllcccccccc}
\hline $\begin{array}{l}\text { Nombre } \\
\text { Común }\end{array}$ & $\begin{array}{l}\text { Nombre } \\
\text { Científico }\end{array}$ & Familia & $\begin{array}{c}\text { \# } \\
\text { Plantas }\end{array}$ & $\begin{array}{c}\text { Altura } \\
(\mathbf{m})\end{array}$ & $\begin{array}{c}\text { Árboles } \\
\text { /ha }\end{array}$ & $\begin{array}{c}\text { DAP } \\
(\mathbf{c m})\end{array}$ & $\begin{array}{c}\text { Área } \\
\text { Basal } \\
\left(\mathbf{m}^{2} / \mathbf{h a}\right)\end{array}$ & AR & DR & IVI \\
\hline Roble & $\begin{array}{l}\text { Quercus } \\
\text { sapoetifolia }\end{array}$ & Fagaceae & 14 & 8 & 70 & 30.7 & 5.19 & 34.15 & 53.34 & 87.48 \\
\hline Encino & $\begin{array}{l}\text { Quercus } \\
\text { segoviensis }\end{array}$ & Fagaceae & 17 & 5 & 85 & 22.5 & 3.38 & 41.46 & 34.67 & 76.13 \\
\hline Nance & $\begin{array}{l}\text { Byrsonima } \\
\text { crassifolia }\end{array}$ & Malpighiaceae & 10 & 5 & 50 & 17.2 & 1.17 & 24.39 & 11.99 & 36.38 \\
\hline Total & & & 409 & & 327 & & 5.00 & 100.00 & 100.00 & 200.00 \\
\hline
\end{tabular}

$\S^{\S}$ Diámetro a la altura del pecho.

Cuadro 8. Número en el mapa (Figura 2), nombre asignado, propietario y hectáreas (área) de los 20 fragmentos de bosque seco identificados en el valle de Agalta, Olancho, Honduras.

\begin{tabular}{cllc}
\hline$\#$ & Nombre & Propietario & Hectáreas \\
\hline 1 & Saguay 1 & Ramón Padilla & 16 \\
\hline 2 & Saguay 2 & Elvin Santos & 9 \\
\hline 3 & Maguelar & María Cruz & 126 \\
\hline 4 & Jimapa & Roberto Chirinos & 16 \\
\hline 5 & Guayacán & Nerlin Turcios, Dionisio Sarmiento y José Víctor Turcios Carias & 410 \\
\hline 6 & Zuntul & Ramiro Turcios & 236 \\
\hline 7 & El Zope & Orlando Cabañas & 27 \\
\hline 8 & Dos ríos & José Manuel Romero Guillén & 19 \\
\hline 9 & Quebracho & Evelio Barahona & 113 \\
\hline 10 & Guajilote 2 & Evelio Barahona & 144 \\
\hline 11 & Callejones & Sabas Oliva & 63 \\
\hline 12 & Guajilote & Evelio Barahona & 72 \\
\hline 13 & Guajilote 3 & Ramón Oliva & 130 \\
\hline 14 & El Barro & Roberto Rubí & 272 \\
\hline 15 & Botadero & Municipalidad de San Esteban/Nora Méndez & 210 \\
\hline 16 & Quebrada Tempisque & José Luis Maldonado, Gilberto Canelas y Erlin Rubí & 92 \\
\hline 17 & Valentín & Manuel Amador & 174 \\
\hline 18 & Carnizuelar 1 & Anselmo Díaz & 50 \\
\hline 19 & Carnizuelar 2 & Carlota Maldonado & 19 \\
\hline 20 & Guajilote 4 & Transito Oliva & 103 \\
\hline & Total & & 2,301 \\
\hline & & & \\
\hline
\end{tabular}


Es un hecho que el bosque seco del valle de Agalta se encuentra seriamente fragmentado por las actividades productivas, pero en aras de proteger el agua se han conservado algunos bosques de galería. Éstos y otros fragmentos remanentes de bosque seco tropical que aún persisten en pequeñas porciones en el valle son de gran importancia. Estas áreas deben tener prioridad para su protección y conservación, ya que representan lo poco que queda de este ecosistema en la zona (Vreugdenhil et al. 2002).

Dicho remanentes del hábitat original en el valle de Agalta consiste en parches de bosque de tamaños varios (Cuadro 8). Son estos remanentes los que albergan a varias especies de fauna, de las cuales varias utilizan esas áreas y las áreas circundantes de hábitat marginal y hasta el agropecuario, particularmente potreros arbolados. Se entiende por hábitat marginal aquellas áreas con vegetación alterada 0 secundaria producto de actividades antrópicas e incluye bordes de vegetación que no se cortó debido por ejemplo al difícil acceso. Además, incluye matorrales, guamiles y áreas de cultivos en barbecho. Los bosques ribereños junto con los cauces de agua proveen la conectividad ecológica básica para el mantenimiento de varias especies de fauna en la zona, incluido posiblemente el colibrí esmeralda hondureño (House 2004).

\section{Literatura Citada}

Anderson, D.L., P. House, R.E. Hyman, R. Steiner, H.R. Hawkins, S. Thorn, M.J. Rey, M.R. Espinal y L.E. Marineros. 2010. Rediscovery of the Honduran Emerald Amazilia luciae in western Honduras: insights on the distribution, ecology, and conservation of a Critically Endangered' hummingbird. Bird Conservation International. BirdLife International. 8 p.

Blake, J.G. y B.A. Loiselle. 2001. Bird assemblages in second-growth and oldgrowth forests, Costa Rica: perspectives from mist nets and point counts. Auk 118: 304-326.

Espinal, M.R. y J.M. Mora. 2012. Estrategia de conservación del colibrí esmeralda (Amazilia luciae) en el valle de Agalta. BID/SOPTRAVI/Dirección General de Carreteras/Cuenta del Desafío del Milenio, Honduras/ICF. Honduras.

Germer, L.D. 2012. Observaciones Puntuales de Colibrí esmeralda Amazilia luciae (Trochilidae) en el Bosque Seco Intermontano del Departamento de Santa Bárbara durante los meses de Abril y Mayo del 2011. El Esmeralda 1:52-64.
Holdridge, L. 1962. Mapa de las zonas de Vida de Honduras. Escala 1:50,000. Tegucigalpa D.C., Honduras.

House, P. 2004. Informe Sobre el Colibrí esmeralda en Quebrada Seca, Arenal. Monitoreo Ecológico Colibrí esmeralda. Unidad de cuencas SANAA. $16 \mathrm{p}$.

INGTELSIG. 2011. Descripción de los Remanentes de Hábitat del Colibrí esmeralda, Departamento de Santa Bárbara, Honduras. SERNA-ICF-EPR. 104 p.

INGTELSIG. 2013. Abundancia, Distribución y Ecología del Colibrí Esmeralda (Amazilia luciae) en el Bosque Seco de Santa Bárbara, Honduras. ICF/SERNA/EPR. Honduras.

Midence, C.M. 2010. Identificación de Áreas Prioritarias para la Conservación de Ecosistemas Semiáridos de Honduras. Tesis de Maestría. Universidad Internacional de Andalucía. $104 \mathrm{p}$.

Nelson, C. 2008. Catálogo de las Plantas Vasculares de Honduras. Secretaria de Recursos Naturales y Ambiente/Editorial Guaymuras. Tegucigalpa. 1,576 p.

Quiel, P. y G. Pineda. 2010. Dinámicas territoriales en Olancho entre los recursos naturales y la agricultura. Informe de la etapa 2A de investigación del Programa de Dinámicas Territoriales Rurales. Rimisp - Centro Latinoamericano para el Desarrollo Rural. Tegucigalpa. $61 \mathrm{p}$.

Reyes, W.M. y A.M. Suazo. 2007. Caracterización biofísica, socioeconómica y ambiental de Saguay y Río Grande, zonas de concentración del teocinte (Dioon mejiae). Departamento de Manejo de Recursos Naturales y Ambiente, Universidad Nacional de Agricultura y Proyecto FNPP, Agenda Forestal Hondureña. Olancho, Honduras.

SERNA (Secretaria de Recursos Naturales y Ambiente). 2008. Especies de Preocupación Especial en Honduras Tegucigalpa, Honduras. 78 p.

Stuart, L.C. 1954. Description of some new amphibians and reptiles from Guatemala. Proceedings of the Biological Society of Washington 67:159-178.

UICN. 2011. Lista roja de especies amenazadas para Honduras según la Union Internacional para la Conservación de la Naturaleza (UICN) VERSIÓN 1. Actualizada enero 2011. 8 p.

Vreugdenhil, D., P.R. House, C.A. Cerrato, R.A. Martínez y A.C. Pereira. 2002. Racionalización del sistema nacional de las Áreas Protegidas de Honduras. Volumen 1: Estudio Principal. PPROBAP/Banco Mundial/UNDP/GEF.

Zúniga E. 1999. Mapa de Clasificación Climática de Honduras. Escala 1:1,000, 000. Tegucigalpa M.D.C., Honduras.

Recibido para publicación el 17 de enero de 2015. Aceptado para publicación el 1 de julio de 2015. 